\title{
Did Evoked Potential Studies Lose Their Significance in the Diagnosis of Multiple Sclerosis?
}

\author{
Gönül Vural*1, Şadiye Gümüşyayla ${ }^{1}$, Hesna Bektaş ${ }^{1}$, Gülsüm Akdeniz ${ }^{2}$ and Orhan Deniz ${ }^{1}$ \\ ${ }^{1}$ Department of Neurology, Yıldırım Beyazıt University, Turkey
}

${ }^{2}$ Department of Biophysics, Electrophysiology Lab, Yildirim Beyazit University, Turkey

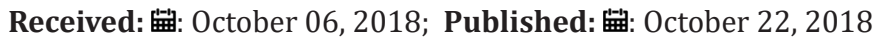

*Corresponding author: Gönül Vural, Yildirim Beyazit University, Medical Faculty, Department of Neurology, Ankara 06800, Turkey

\section{Abstract}

Objective: Since magnetic resonance imaging (MRI) became central in the diagnosis and follow-up of Multiple Sclerosis (MS), evoked potentials (EP) studies appear to have lost their value, despite their ability to indicate the real-time status of the physiological system, and the nature of the lesion. In this study, we investigated the diagnostic utility of EPs and their correlation with MRI findings in our patients who were evaluated for suspected MS.

Materials and Methods: In this study, the medical records at first admission of 100 patients who were evaluated for suspected MS were analyzed retrospectively. The correlations of EPs with clinical and MRI findings were analyzed.

Results: The VEP test was more sensitive than orbital MRI in detecting optic nerve involvement. tSEP agreed with $81 \%$ of the MRI findings and mSEP with 55\%. On the other hand, in patients with normal spinal MRI, mSEP and tSEP indicated functional impairment in $44 \%$ and $67 \%$ of the cases, respectively. Although MRI seems to be more useful in detecting brainstem abnormalities, only the BAEP study revealed subclinical involvement in $12 \%$ of the patients. Again, VEP indicated subclinical involvement in $18 \%$ and $\mathrm{mSEP}$ in $29 \%$ of the cases.

Discussion: Evoked potential studies are still valuable in revealing abnormalities that cannot be detected by conventional MRI techniques. It is an indisputable fact that EP is more sensitive than MRI in detecting optic nerve lesions. In particular, EPs are especially useful for diagnosis and follow-up when MRI cannot be performed or takes too much time.

Keywords: Evoked Potentials; Multiple Sclerosis; Magnetic Resonance Imaging

Abbreviations: MRI: Magnetic Resonance Imaging; MS: Multiple Sclerosis; EP: Evoked Potentials; VEP: Visual Evoked Potential

\section{Introduction}

Since magnetic resonance imaging (MRI) became central in the diagnosis and follow-up of Multiple Sclerosis (MS), evoked potentials (EP) studies appear to have lost their value, despite their ability to indicate the real-time status of the physiological system, and the nature of the lesion [1-5]. Indeed, EPs are rarely mentioned in the current literature, and are not found as frequently as MRI in clinical studies. The measurement of EPs is noninvasive, inexpensive, easy to access and implement, and provides clear numerical values to evaluate signal propagation in time and space in cases of suspected MS [6]. In this study, we investigated the diagnostic utility of EPs and their correlation with MRI findings in our patients who were evaluated for suspected MS.

\section{Materials and Methods}

In this study, the medical records at first admission of 100 patients who were evaluated for suspected MS were analyzed retrospectively. The correlations of evoked potentials with clinical and MRI findings were analyzed.

The EPs tests included: visual evoked potential (VEP), median nerve somatosensory evoked potential (median SEP), tibial nerve somatosensory evoked potential (tibial SEP), and brainstem auditory evoked potential (BAEP). A Keypoint (Alpine, Denmark) instrument was used to record the EPs. All MRI images were obtained with a $1.5 \mathrm{~T}$ super-conductive magnet (Philips Achieva) with a standard head coil of 8-16 channels. Transverse T1 and T2weighted, transverse FLAIR, Coronal T2-weighted, and contrast T1 sequences were obtained, according to the conventional MRI protocol.

\section{Statistics}

The sensitivity of the methods was compared using Fisher's exact test. The sensitivity of the tests was calculated as the fraction 
of abnormal findings in patients diagnosed with MS according to McDonald criteria. $p<0.05$ and $p<0.01$ were considered statistically significant.

\section{Results}

The records at first admission of 32 male and 68 female patients were examined. The age range was 22-34 years (mean 26.7) for male patients, and 18-42 (mean 25.2) for females. All patients underwent cranial MRI examination. Moreover, 16 patients had orbital MRI, 93 patients had cervical MRI and 23 patients underwent thoracic MRI. VEP was recorded on all patients, median SEP (mSEP) on 93 of them, tibial SEP (tSEP) on 21 and BAEP on 92. The mean P100 wave latency was prolonged in 15 out of 16 symptomatic patients with orbital MRI. Only 5 of these patients showed orbital MRI lesions. In the other 10 patients, while the orbital MRI was normal, the P100 wave latency was prolonged (Table 1$)$. In 72 patients $(72 \%)$, VEP examination showed delayed conduction. Of these, 54 were symptomatic and 18 were asymptomatic. Out of 28 patients with normal VEP examination, 4 had visual symptoms (Table 2). Forty patients out of the 93 who had mSEP performed were symptomatic. Out of the 28 patients found abnormal by both spinal MRI and mSEP examination, 9 were subclinical.

Table 1: Optic nerve abnormalities on MRI and VEP.

\begin{tabular}{|c|c|}
\hline Data & $\mathbf{n = 1 6}$ \\
\hline Abnormal orbital MRI; abnormal VEP & 5 \\
\hline Abnormal orbital MRI; normal VEP & 0 \\
\hline Normal orbital MRI; abnormal VEP & 10 \\
\hline Normal orbital MRI; normal VEP & 1 \\
\hline
\end{tabular}

Table 2: VEP abnormalities in patients with MS.

\begin{tabular}{|c|c|}
\hline Data & n =100 \\
\hline with symptom; abnormal VEP & 54 \\
\hline with symptom; normal VEP & 4 \\
\hline without symptom; abnormal VEP & 18 \\
\hline without symptom; normal VEP & 24 \\
\hline
\end{tabular}

In 24 patients spinal MRI revealed a cervical lesion while mSEP was normal. Three of these patients were subclinical. In 18 asymptomatic patients the mSEP was delayed while spinal MRI was normal, therefore mSEP was the only evidence of subclinical involvement. Spinal MRI alone indicated subclinical involvement in only 3 patients (Table 3). Complete spinal MRI (cervical and thoracic) was performed on 23 patients. tSEP examination was performed on 21 out of 23 fully symptomatic patients and conduction delay was detected in 18 of them. In 16 of these, spinal MRI revealed lesions, while it was normal in the other 2 (Table 4). mSEP was abnormal in 46 (49\%) of 93 tested patients, and in 18 (86\%) of 21 patients who were tested for tSEP. Agreement of MRI findings with tSEP and was found in $81 \%$ of the cases, and in $55 \%$ with mSEP. BAEP was performed on 92 patients and abnormalities were detected in 12 of them (13\%). Only one of these patients was symptomatic. In 9 out of 12 patients with BAEP interpeak latency delay, an MRI lesion was detected in the appropriate localization, while 3 had no MRI localized lesions.
Table 3: The agreement between MRI and mSEP.

\begin{tabular}{|c|c|}
\hline Data & $\mathrm{n}=93$ \\
\hline $\begin{array}{l}\text { Abnormal cervical MRI; abnormal median } \\
\text { SEP }\end{array}$ & $\begin{array}{l}28 \text { (19 symptomatic, } 9 \\
\text { subclinical) }\end{array}$ \\
\hline $\begin{array}{l}\text { Abnormal cervical MRI; normal median } \\
\text { SEP }\end{array}$ & $\begin{array}{l}24 \text { (21 symptomatic, } 3 \\
\text { subclinical) }\end{array}$ \\
\hline $\begin{array}{l}\text { Normal cervical MRI; abnormal median } \\
\text { SEP }\end{array}$ & 18 (all subclinical) \\
\hline Normal cervical MRI; normal median SEP & 23 (all subclinical) \\
\hline
\end{tabular}

Table 4: The agreement between MRI and tSEP.

\begin{tabular}{|c|c|}
\hline Data & $\mathbf{n = 2 3}$ \\
\hline Abnormal spinal MRI; abnormal tibial SEP & 16 \\
\hline Abnormal spinal MRI; normal tibial SEP & 2 \\
\hline Normal spinal MRI; abnormal tibial SEP & 2 \\
\hline Normal spinal MRI; normal tibial SEP & 1 \\
\hline Abnormal spinal MRI; tibial SEP not applied & 1 \\
\hline Normal spinal MRI; tibial SEP not applied & 1 \\
\hline
\end{tabular}

Although 37 patients had brain stem lesions on cranial MRI, BAEP was normal in 28 of them (Table 5). In 33 examinations (21 asymptomatic and 12 symptomatic) the MRI of the related region did not detect lesions, whereas EPs (VEP, medial and tibial SEP, BAEP) detected abnormalities (Table 6). Moreover, considering the fact that only the VEP test revealed subclinical optic nerve involvement in 18 patients who were asymptomatic and who had not undergone orbital MRI examination, subclinical effects were detected only by EP tests in a total of 39 cases. VEP was abnormal in $72(72 \%)$ of the 100 patients tested, in 46 (49\%) of 93 patients tested for mSEP, in $18(86 \%)$ of the 21 tested for tSEP, and in 12 $(13 \%)$ of the 92 patients tested for BAEP (Figure 1). When these four types of EPs test were compared, VEP, mSEP and tSEP gave significantly more abnormal results than BAEP in MS patients. VEP and tSEP abnormality rates were comparable. Both VEP and tSEP showed a higher percentage of abnormalities than mSEP (Table 7). A pathway delay was revealed by VEP in 10 out of 11 (91\%) patients with negative orbital MRI, by mSEP in 18 out of $41 \mathrm{MRI}$ negative patients (44\%), by tSEP in 2 out of $3(67 \%)$ MRI-negative patients, and by BAEP in 3 out of 55 (5\%) MRI-negative patients (Figure 2).

Table 5: The agreement between MRI and BAEP.

\begin{tabular}{|c|c|}
\hline Data & $\mathbf{n = 9 2 ~ ( 1 ~ s y m p t o m a t i c ) ~}$ \\
\hline Abnormal brainstem MRI; abnormal BAEP & 9 (1 symptomatic) \\
\hline Abnormal brainstem MRI; normal BAEP & 28 \\
\hline Normal brainstem MRI; abnormal BAEP & 3 \\
\hline Normal brainstem MRI; normal BAEP & 52 \\
\hline Abnormal spinal MRI; tibial SEP not applied & 1 \\
\hline Normal spinal MRI; tibial SEP not applied & 1 \\
\hline
\end{tabular}


Table 6: EP abnormalities in MRI-negative MS patients for related pathway.

\begin{tabular}{|c|c|}
\hline Data & \\
\hline Abnormal VEP; normal MRI & 10 (all symptomatic) \\
\hline Abnormal median SEP; normal MRI & 18 (all asymptomatic) \\
\hline Abnormal tibial SEP; normal MRI & 2 (all symptomatic) \\
\hline Abnormal BAEP; normal MRI & 3 (all asymptomatic) \\
\hline
\end{tabular}

Table 7: Statistical significance for the difference between the modalities of evoked potentials.

\begin{tabular}{|c|c|c|c|}
\hline Parameters & VEP & mSEP & tSEP \\
\hline VEP & & & \\
\hline mSEP & $0.002^{* *}$ & & \\
\hline tSEP & 0.273 & $0.003^{* *}$ & \\
\hline BAEP & $0.000^{* *}$ & $0.000^{* *}$ & $0.000^{* *}$ \\
\hline \multicolumn{4}{|c|}{ Fisher's exact test ${ }^{*} \mathrm{p}<0.05 .{ }^{* *} \mathrm{p}<0.01$} \\
\hline
\end{tabular}

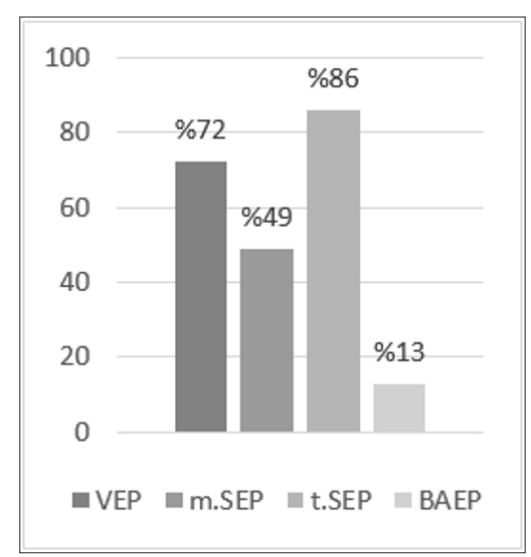

Figure 1: Percentage of abnormal results of VEP, mSEP, tSEP and BAEP in patients with MS.

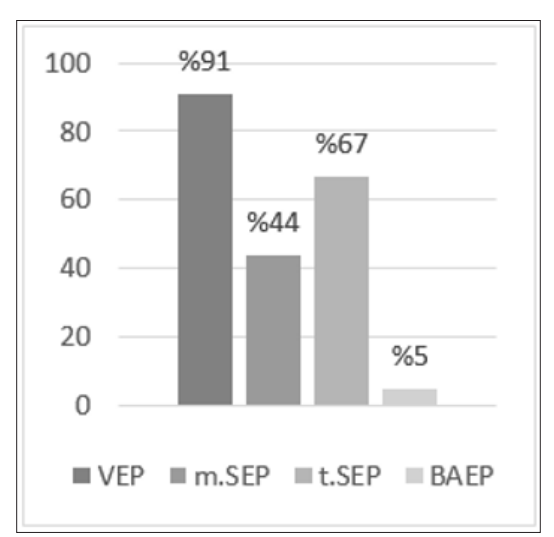

Figure 2: Percentage of abnormal results of VEP, mSEP, tSEP and BAEP in MRI-negative MS patients for related pathway.

When there was no visible lesion in the MRI of the relevant region, VEP was more sensitive than $\mathrm{MSEP}$ and BAEP, but not than tSEP, in revealing the abnormality in the related pathway. tSEP and mSEP were more sensitive than BAEP, and their sensitivity was comparable (Table 8). VEP in 18 out of 100 (18\%) patients tested, mSEP in 27/93 (29\%), and BAEP in 11/92 (12\%) showed evidence of subclinical involvement in asymptomatic patients (Figure 3). Thus, mSEP is superior to BAEP in detecting subclinical involvement and the sensitivity of VEP is similar to that of BAEP and mSEP (Table 9).

Table 8: Statistical significance for the difference between the modalities of evoked potentials in MRI-negative MS patients for related pathway.

\begin{tabular}{|c|c|c|c|}
\hline Parameters & VEP & mSEP & tSEP \\
\hline VEP & & & \\
\hline mSEP & $0.007^{* *}$ & & \\
\hline tSEP & 0.396 & 0.583 & \\
\hline BAEP & $0.000^{* *}$ & $0.000^{* *}$ & $0.018^{*}$ \\
\hline \multicolumn{4}{|c|}{ Fisher's exact test ${ }^{*} \mathrm{p}<0.05 .^{* *} \mathrm{p}<0.01$} \\
\hline
\end{tabular}

Table 9: Statistical significance for the difference between the modalities of evoked potentials in asymptomatic MS patients for related pathway.

\begin{tabular}{|c|c|c|}
\hline Parameters & VEP & mSEP \\
\hline VEP & & \\
\hline mSEP & 0.088 & \\
\hline BAEP & 0.314 & $0.006^{* *}$ \\
\hline \multicolumn{2}{|c|}{ Fisher's exact test ${ }^{*} \mathrm{p}<0.05{ }^{* *} \mathrm{p}<0.01$} \\
\hline
\end{tabular}

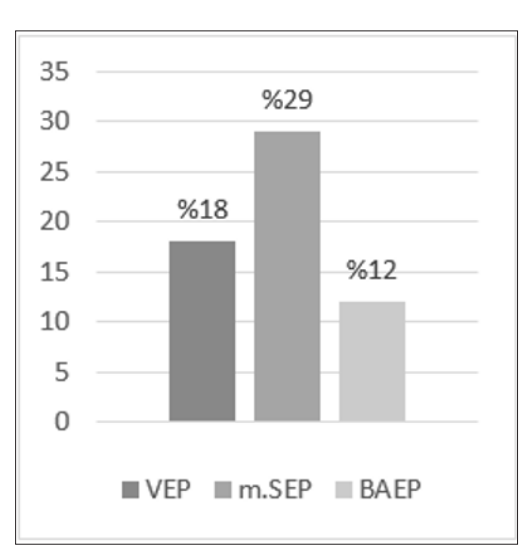

Figure 3: Percentage of abnormal results of VEP, mSEP and BAEP in asymptomatic MS patients.

\section{Discussion}

Abnormal VEP, mSEP, tSEP, and BAEP results were found, respectively, in $72 \%, 49 \%, 86 \%$, and $13 \%$ of our patients. The VEP test was more sensitive than orbital MRI in detecting optic nerve involvement. Moreover, in all patients in whom orbital MRI demonstrated optic nerve involvement, VEP had already detected a delay in conduction. Orbital MRI did not provide additional information about optic nerve involvement in patients who had normal VEP. tSEP was more often abnormal than mSEP, but this may be due to the fact that tSEP was performed only on symptomatic patients. tSEP agreed with $81 \%$ of the MRI findings 
and mSEP with 55\%. On the other hand, in patients with normal spinal MRI, mSEP and tSEP indicated functional impairment in $44 \%$ and $67 \%$ of the cases, respectively. Although MRI seems to be more useful in detecting brainstem abnormalities, in 3 patients, only the BAEP study revealed subclinical involvement. Again, VEP indicated subclinical involvement in 18\% and mSEP in 29\% of the cases. Most of the clinical symptoms of MS reflect altered impulse generation and transmission in the central nervous system.

EPs are measurements of central signal transduction in vivo and provide a quantitative measurement of functional impairment in the associated pathways [2-5]. After Halliday et al. [7] reported a delay in P100 wave latency in almost all MS patients, even with no history of optic neuritis, VEP tests attracted great interest: Many later studies repeatedly showed similar findings, although with smaller effects than reported in the original one, thus establishing VEP as a sensitive method to detect clinically silent lesions. In later studies, a significant number of patients with MS were also shown to have BAEP and SEP abnormalities without a lesion in the relevant site [8-10]. Before the advent of MRI, EPs were a routine component of the diagnosis of MS. However, due to its relatively high specificity and sensitivity to subclinical lesions, for several years MRI has largely replaced EPs in monitoring the dissemination of lesions in time and space in patients presenting with the typical symptoms of MS and excluding alternative diagnoses [1]. However, overconfidence in imaging results leads to misdiagnosis. In spite of well-established diagnostic criteria, misdiagnosis remains a problem.

In a study conducted by Solomon et al., the final diagnoses of 110 patients with MS who were re-evaluated in a MS center was changed to migraine (22\%), fibromyalgia (15\%), nonspecific symptoms with abnormal MRI (12\%), psychogenic disorders (11\%), and neuromyelitis optica spectrum disorders (6\%). In these cases, cerebrospinal fluid analysis and EPs can help the clinician [11]. Although current criteria for MS diagnosis do not require neurophysiological testing [1], there are cases in which electrophysiological data can provide useful diagnostic information. EPs may show spatial dissemination of the lesions. During the course of MS, EPs may be used to confirm uncertain relapses in patients expressing vague or transient symptoms [12]. It has been reported that $50-90 \%$ of MS patients have abnormalities in one or more of the EPs [13]. The ability of EPs to detect subclinical lesions of the optic nerve and spinal cord has been demonstrated in many studies [14]. Although conventional MRI is accepted as the standard examination in diagnosing MS and monitoring the disease, it usually shows weak association with functional data, a phenomenon known as functional-anatomical paradox $[5,15]$.

In contrast, EP can be used to assess the long, relevant pathways that are well correlated with functional involvement [16]. The sensitivity of an EP study depends on the length of the measured pathways and the probability that the relevant functional system suffers a demyelinating lesion [4]. Ko [17] showed that VEP can detect optic nerve lesions with 100\% sensitivity whereas SEP has lower sensitivity (64\%) in detecting spinal cord lesions. This result is in contrast with the expectation that long afferent spinal tracts are more common in MS, because the SEP will be normal in small cord lesions that leave a sufficient number of fast conducting fibers intact. This study also revealed the near equality of BAEP and MRI in detecting brain stem lesions, the superiority of MRI over SEP in detecting spinal cord lesions, and the undisputed superiority of VEP over MRI in detecting optic nerve lesions. In their study, Dijuric et al. [18] found that tSEP was more sensitive than mSEP, VEP and BAEP, followed by VEP. BAEP had the lowest sensitivity. Although EPs can detect subclinical CNS involvement, their sensitivity to brain lesions is much lower than MRI.

MRI is clearly superior to EPs in detecting supratentorial lesions, since plaques are preferably located in periventricular regions, which do not affect sensory and motor pathways, while EPs can only be abnormal when such pathways are affected [19]. Though EPs provide information limited to known pathways, and this fact limits their value in the diagnosis of MS, this is an advantage when evaluating functional impairments in MS patients, since EPs are directly related to functionality $[19,20]$. Although it is now easier to diagnose MS with the new MRI-based diagnostic criteria, many aspects of the disease can be demonstrated primarily or only by using neurophysiological studies that today appear to be generally neglected. The expert consensus of the MAGNIMS network suggested the inclusion of optic nerve involvement for diagnosing spatial dissemination in the revised McDonald's criteria [21]. What does EP tell us? A normal EP test when MRI shows demonstrable involvement of the related pathways tells us that an adequate amount of fast-conducting fibers is still retained [17].

Furthermore, if despite a delayed initial response in EP the cortical response occurs within the normal latency range, we may infer that functional amplification and reorganization are still satisfactory [22-25]. In contrast, EPs cannot capture silent lesions located outside the relevant pathways. Since there are no relevant pathways that can be evaluated with EPs in the periventricular field, which is frequently affected in MS, EPs will not provide information about this region [19]. Because of these handicaps, a normal EP result (SEP and BAEP) cannot guarantee the preserved functionality of related pathways. But, an EP abnormality can tell us, in the early stages of the disease, that the normal-looking white matter in the MRI of the relevant pathway is not actually normal. EP abnormalities, irrespective of the presence of visible lesions in the MRI, are never negligible, as they can reveal both the inadequacy of fast-transmitting fibers and a serious functional impact with insufficient adaptation. So EPs tell us more than we think. In cases where MRI and neurological examination and anamnesis provide only the minimum conditions for diagnosis, subclinical abnormalities shown in one or several EPs will also be effective as additional parameters to devise a treatment plan.

\section{Conclusion}

It is clear that evoked potential studies are still valuable in revealing abnormalities that cannot be detected by conventional MRI techniques. It is an indisputable fact that EP is more sensitive 
than MRI in detecting optic nerve lesions. In particular, EPs are especially useful for diagnosis and follow-up when MRI cannot be performed, or takes too much time.

\section{References}

1. Polman CH, Reingold SC, Banwell B, Clanet M, Cohen JA, et al. (2011) Diagnostic criteria for multiple sclerosis: 2010 revisions to the McDonald criteria. Ann Neurol 69(2): 292-302.

2. Waxman SG (1988) Clinical course and electrophysiology of multiple sclerosis. In: Waxman SG (Ed.) Advances in neurology, functional recovery in neurological disease. Raven Press, New York, USA 47: 157184.

3. Smith KJ (1994) Conduction properties of central demyelinated and remyelinated axons, and their relation to symptom production in demyelinating disorders. Eye (Lond) 8: 224-237.

4. Hardmeier M, Leocani L, Fuhr P (2017) A new role for evoked potentials in MS? Repurposing evoked potentials as biomarkers for clinical trials in MS. Mult Scler 23(10): 1309-1319.

5. Giffroy X, Maes N, Albert A, Maquet P, Crielaard JM, et al. (2016) Multimodal evoked potentials for functional quantification and prognosis in multiple sclerosis. BMC Neurology 16: 83.

6. Brooks JB, Jardim MR, Papais-Alvarenga RM, Fragoso YD (2015) There is still a role for the blink reflex in the diagnosis and follow-up of multiple sclerosis. Clin Neurophysiol 126 (4): 743-747.

7. Halliday AM, Mc Donald WI, Mushin J (1973) Visual evoked response in diagnosis of Multiple Sclerosis. Br Med J 15(4): 661-664.

8. Asselman P, Chadwick DW, Marsden DC (1975) Visual evoked responses in the diagnosis and management of patients suspected of multiple sclerosis. Brain 98(2): 261-282.

9. Trojaborg W, Petersen E (1979) Visual and somatosensory evoked cortical potentials in multiple sclerosis. J Neurol Neurosurg Psychiatry 42(4): 323-330.

10. Kjaer M (1980) The value of brain stem auditory, visual and somatosensory evoked potentials and blink reflexes in the diagnosis of multiple sclerosis. Acta Neurol Scand 62(4): 220-236.

11. Solomon AJ, Bourdette DN, Cross AH, Applebee A, Skidd PM, et al. (2016) The contemporary spectrum of multiple sclerosis misdiagnosis: A multicenter study. Neurology 87(3): 1393-1399.

\section{ISSN: 2574-1241}

DOI: 10.26717/BJSTR.2018.10.001915

Gönül Vural. Biomed J Sci \& Tech Res

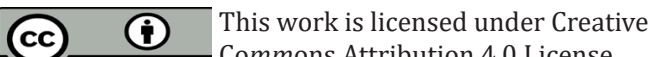
Commons Attribution 4.0 License

Submission Link: https://biomedres.us/submit-manuscript.php
12. Comi G, Leocani L, Medaglini S, Locatelli T, Martinelli V, et al. (1999) Measuring Evoked Responses in Multiple Sclerosis. Mult Scler 5(4): 263267.

13. Adams RD, Victor M, Ropper AH (2001) Multiple sclerosis and allied demyelinative diseases. In: Wonsiewicz MJ, Medina MP, Navzorov M (Eds.). Principles of neurology ( $7^{\text {th }}$ edn.). McGraw- Hill, New York, USA pp. 954-982.

14. Chiappa KH (1997) Evoked potentials in clinical medicine ( $3^{\text {rd }}$ edn). Lippincott-Raven, Philadelphia pp. 95-401.

15. Zivadinov R, Leist TP (2005) Clinical-magnetic resonance imaging correlations in multiple sclerosis. J Neuroimaging 15(4): 10-21.

16. Leocani L, Comi G (2008) Neurophysiological Markers. Neurol Sci 29(2): 218-221.

17. Ko KF (2010) The role of evoked potential and MR imaging in assessing multiple sclerosis: A comparative study. Singapore Med J 51(9): 716-720.

18. Djuric S, Djuric V, Zivkovic M, Milosevic V, Jolic M, et al. (2010) Are somatosensory evoked potentials of the tibial nerve the most sensitive test in diagnosing multiple sclerosis? Neurol India 58(4): 537-541.

19. Leocani L, Comi G (2014) Clinical neurophysiology of multiple sclerosis. In: Goodin DS (Ed) Multiple Sclerosis and Related Disorders, Handbook of Clinical Neurology ( $3^{\text {rd }}$ series), Elsevier, Amsterdam 122: 671-79.

20. Leocani L, Comi G (2000) Neurophysiological investigations in multiple sclerosis. Curr Opin Neurol 13: 255-261.

21. Filippi M, Rocca MA, Ciccarelli O, De Stefano N, Evangelou N, et al. (2016) MRI criteria for the diagnosis of multiple sclerosis: MAGNIMS consensus guidelines. Lancet Neurol 15:292-303.

22. Seyal M, Gabor AJ (1987) Generators of human spinal somatosensory evoked potentials. J Clin Neurophysiol 4(2): 177-187.

23. Urasaki E, Wada S, Kadoya C, Matsuzaki H, Yokota A, et al. (1988) Absence ofspinal N13-P13 and normal scalp far-field P14 in a patient with syringomyelia. Electroencephalogr Clin Neurophysiol 71: 400-404.

24. Desmedt JE, Cheron G (1980) Central somatosensory conduction in man: neural generators and interpeak latencies of the far-field components recorded fromneck and right or left scalp and earlobes. Electroencephalogr Clin Neurophysiol 50(5-6): 382-403.

25. Eisen A, Purves S, Hoirch M (1982) Central nervous system amplification: its potential in the diagnosis of early multiple sclerosis. Neurology 32(4): 359-364.

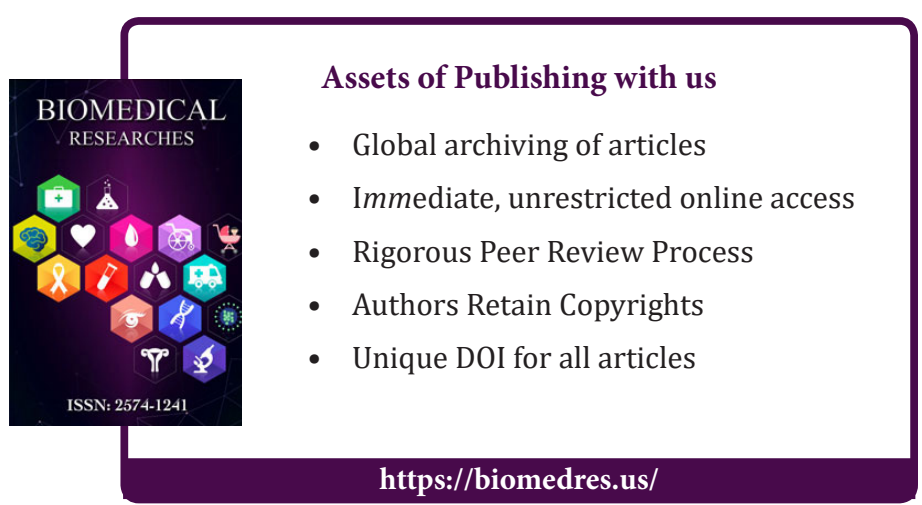

